\title{
Developmental Changes of Mechanics Measured in the Gerbil Cochlea
}

\author{
Gulam Emadi $^{1,3}$ and Claus-Peter Richter ${ }^{1,2,3}$ \\ ${ }^{1}$ Department of Biomedical Engineering, Northwestern University, Evanston, IL 60208, USA \\ ${ }^{2}$ Department of Otolaryngology Head-and Neck Surgery, Northwestern University Feinberg School of Medicine, \\ Chicago, IL, USA \\ ${ }^{3}$ Auditory Physiology Laboratory (The Hugh Knowles Center), Department of Communication Sciences and Disorders, \\ Northwestern University, Evanston, IL, USA
}

Received: 7 November 2005; Accepted: 2 October 2007; Online publication: 29 November 2007

\begin{abstract}
This report describes stiffness and best frequency measurements obtained in vitro from the basilar membrane of the gerbil cochlea at the onset of hearing, during hearing maturation, and after hearing has matured. Our stiffness data constitute the first direct experimental evidence of developmental stiffness changes in the basal and middle turns. Stiffness changes by a factor of 5.5 in the basal turn between postnatal day 11 and adult, and the difference from adult is statistically significant for all ages measured up to postnatal day 16 . For the middle turn, stiffness changes by a factor of 1.6 between postnatal day 11 and adult. Whereas for postnatal day 12 and beyond there is no statistically significant difference from adult, our data suggest that there may be a significant difference of stiffness between day 11 and adult in the middle turn. For the basal turn, our motion measurements confirm a passive component to the developmental best frequency shift. For the middle turn, changes in best frequency are not statistically significant. Best frequency was determined by stimulating the tissue at audio frequencies with a glass paddle and measuring motion with a computer-based imaging system. Tissue stiffness was measured with a piezoelectric-based sensor system. Tissue stiffness changes have previously been postulated to contribute to the best frequency
\end{abstract}

Correspondence to: Claus-Peter Richter - Department of Otolaryngology Head- and Neck Surgery - Northwestern University Feinberg School of Medicine - Searle Building 12-561, 303 East Chicago Avenue, Chicago, IL 60611-3008, USA. Telephone: +1-312-5031603; fax: +1-312-5031616; email: cri529@northwestern.edu shift observed in the cochlear base. Incorporating our data into a simple spring-mass resonance model demonstrates that our experimentally measured stiffness change can account for the change of best frequency. These results suggest that a stiffness change is, in fact, a critical component of the best frequency shift observed in the basal turn of the gerbil cochlea after the onset of hearing.

Keywords: cochlea, mechanics, development, stiffness, basilar membrane, best frequency

\section{INTRODUCTION}

In this report, we provide direct measurements of basilar membrane stiffness and best frequency $(\mathrm{BF})$ obtained during the course of hearing onset and maturation in the gerbil cochlea. In a previous study (Emadi et al. 2004), basilar membrane stiffness changes were shown to account for BF changes along the length of the adult gerbil cochlea. In this report, we again examine the correlation between basilar membrane stiffness and BF, but our analysis here is based on comparing measurements obtained at fixed locations (one in the basal turn and one in the middle turn) across multiple developmental time points. The gerbil is well suited for utilizing developmental time courses to gain insight into structure-function relationships because its cochlea undergoes significant anatomical and functional development during the first few weeks postnatal. No sound-evoked electrophysiologi- 
cal responses can be elicited from the gerbil cochlea until 12 days after birth (Harris and Dallos 1984; Woolf and Ryan 1984; Echteler et al. 1989). The best frequencies measured electrophysiologically in basal turn regions shift upward by approximately 1.5 octaves between 12 and 18 days postnatal, and adult-like frequency responses are not evident in these regions until approximately 18 days after birth (Harris and Dallos 1984; Woolf and Ryan 1984; Yancey and Dallos 1985; Arjmand et al. 1988; Echteler et al. 1989; McGuirt et al. 1995; Mills and Rubel 1996, 1997, 1998; Overstreet et al. 2002). Beyond 18 days, only subtle maturation of cochlear function can be detected (McGuirt et al. 1995; Overstreet et al. 2003). Middle turn regions, on the other hand, do not exhibit any such developmental shift of best frequency between the time of hearing onset and maturation (Arjmand et al. 1988; Müller 1996; Richter and Dallos 2000).

It has been suggested that the upward shift of best frequency observed in the basal turn may be caused by concomitant tissue stiffness changes, which have been deduced from anatomical development (Echteler 1995; Schweitzer et al. 1996). We have examined this contention experimentally by measuring stiffness and BF during development in the gerbil hemicochlea, a preparation that has already been used successfully for the measurement of anatomical development (Richter et al. 2000). The present experiments reveal significant developmental increases of stiffness in the basal and middle turns. By incorporating the stiffness values and previously measured cochlear dimensional data into a simple mechanical model, we confirm that developmental basilar membrane stiffness changes can quantitatively account for the bulk of the observed developmental BF changes. This relationship between stiffness and $\mathrm{BF}$ is consistent with our previous demonstration that, in the adult, longitudinal stiffness gradients can account for the frequency gradient along the length of the gerbil cochlea (Emadi et al. 2004). Portions of this work have been presented in conference proceedings (Richter and Dallos 2000; Emadi et al. 2001).

\section{METHODS}

All animal experiments followed guidelines from the National Institutes of Health and the National Science Foundation and were approved by the Northwestern University Animal Care and Use Committee.

\section{Hemicochlea preparation}

Hemicochleae were prepared as described in previous studies (Hu et al. 1995; Edge et al. 1998; Richter et al. 1998; Hu et al. 1999; Emadi et al. 2004; Teudt and
Richter 2007). In brief, chloroform-sedated gerbils (Meriones unguiculatus) of various developmental ages were anesthetized with sodium pentobarbital $(180 \mathrm{mg} / \mathrm{kg}$ body weight) and killed by rapid cervical dislocation followed immediately by decapitation. The head was divided in the midsaggital plane, and the bullae were extracted and placed in oxygenated bathing solution (adjusted to $\mathrm{pH}$ 7.3-7.4 and 310-317 mOsm) containing the following (in $\mathrm{mM}$ ): $5 \mathrm{KCl}, 10$ HEPES, $45 \mathrm{NaCl}$, $105 \mathrm{NaOH}$, and 100 lactobionic acid. The primary purpose of this bath formulation was to minimize mechanical tissue distortion up to at least 2 hours postmortem; a more detailed justification of the use of this solution has been published (Emadi et al. 2004). After exposing the cochlea, each bulla was glued to a metal block using cyanoacrylate and then the cochlea was cut with a vibratome into two parts along a plane containing its modiolus. One of the resulting hemicochleae was positioned in a petri dish containing the bathing medium and was oriented to yield a crosssectional view of the tissues in a selected cochlear turn. For inclusion in our analysis, a hemicochlea had to exhibit the following features: straight inner and outer pillar cells, long cylindrical outer hair cells, no bend along the basilar membrane upper margin, and a tectorial membrane closely apposed to the reticular lamina. The most sensitive indicator of deterioration of the preparation was a separation of the tips of the third row outer hair cell stereocilia from the tectorial membrane and a separation of the tips of the inner hair cell stereocilia from Hensen's stripe.

The hemicochlea preparation is a versatile tool for studying micromechanics at multiple well-defined locations along the cochlear spiral. Because of the relatively weak mechanical coupling along the longitudinal dimension of the basilar membrane (Richter et al. 1998; Naidu and Mountain 2001; Emadi et al. 2004), a given radial cross-section can be studied as a semi-independent entity. In support of the mechanical integrity of a hemicochlea, we apply multiple lines of evidence including morphology (as described above), similarity of in vivo and in vitro stiffness measurements, and stability of stiffness measurements pre- and post-mortem (Emadi et al. 2004).

Nevertheless, as addressed in earlier work (e.g., Emadi et al. 2004), we recognize that interpretation of results with the hemicochlea must be made with care. Two issues in particular are of relevance for the present paper. First, the scalae are acoustically shunted, which means that normal in-vivo-like pressure waves cannot be applied across the cochlear partition. Fortunately, local mechanical stimulation of the basilar membrane has been demonstrated to produce motion patterns and frequency response characteristics similar to those elicited in vivo with acoustic stimulation (Richter et al. 1998). Second, the 
electrical environment of the hemicochlea is altered relative to that in vivo, namely, the hair cell resting potentials are reduced (because of leakage current and rundown of ATP-dependent pumping activity) and the endocochlear potential (EP) is eliminated (because of shunting of the scalae and the absence of a blood supply to the stria vascularis). Under normal conditions, these combined potentials drive transduction current through the hair cells in vivo. Elimination of the EP alone is sufficient to compromise the sharp tuning and high sensitivity observed in the normal "active" cochlea (Ruggero and Rich 1991). We contend that, although the hemicochlea is not the ideal tool for studying the active mechanics of the cochlea, it is nonetheless suitable for the present study, which examines the passive mechanics within localized cross-sections of the cochlea.

\section{Measurement of best frequency (imaging system)}

The hemicochlea preparation makes it relatively easy to view the structures within a radial cross-section of the cochlear partition and to make simultaneous measurements of the dynamic motion at many points within this cross-section. Although it is possible to obtain an equivalent set of motion data by making many sequential linear measurements from multiple angles (e.g., using confocal laser interferometry), it is far simpler to obtain such data array using an imaging system in conjunction with the hemicochlea. The system for measuring cochlear motions has been described previously in a book chapter (Richter and Dallos 2003) and will be discussed here in detail. The overall procedure can be summarized as follows: cochlear tissues mechanically stimulated across a range of audio frequencies, images of the resulting motion at each frequency were captured with a stroboscopic video system, a software algorithm ( $\mathrm{Hu}$ et al. 1999; Cai et al. 2003) was applied off-line to these images to quantify tissue displacement for chosen structures in a cochlear cross-section, and a frequency response curve was constructed to determine the best frequency $(\mathrm{BF})$ of motion for each of these structures.

\section{Measurement procedure}

The video system included a microscope (Leitz Medilux) equipped with water-immersion objectives (Olympus $10 \times, 20 \times$, and $40 \times$ ), an Optivar $(1-2 \times)$, a trinocular viewing head, a CCD camera (Panasonic GP-MF602), and an LED light source arranged below the microscope's condenser. The LEDs were positioned off the optical axis so that the preparation could be illuminated obliquely to increase the contrast of the relatively translucent tissues of the cochlea; using the LEDs had the additional advan- tage of allowing for high frequency strobing of the light during data acquisition. A hemicochlea was affixed in a petri dish using high-vacuum grease, fully immersed in bath solution, placed on the stage of the microscope, and oriented to image a single turn into the camera. The cochlear tissues were then locally driven at 15 chosen audio frequencies between 100 and 5,000 $\mathrm{Hz}$ for the middle turn and between 1,000 and $16,000 \mathrm{~Hz}$ for the basal turn. This stimulation was applied using a glass paddle, which was a 2-mm pipette pulled and sealed to a tip diameter of approximately $100 \mu \mathrm{m}$ and mounted to a piezoelectric bimorph driven by a precision sinusoidal waveform generator (Hewlett Packard 33120A). The piezo bimorph allowed the application of sinusoidal stimuli up to at least $20 \mathrm{kHz}$. Based on the assumptions that the hemicochlea is a passive preparation and that its mechanical response should have a linear amplitude dependence, we contend that the shape of the frequency response should not depend on the distance between the paddle and the tissue (i.e., on the overall stimulus level). Nevertheless, as an additional precaution, efforts were made to place the paddle at a consistent distance (observed visually) of approximately $30 \mu \mathrm{m}$ from the basilar membrane across experiments. The LED light source was synchronously strobed to yield an image that was "frozen" at a particular phase of the tissue motion. In this way, static images could be acquired and averaged by the video system at multiple fixed stimulus phases-for each stimulus frequency, 18 images at time points spaced evenly throughout the cycle of motion (i.e., $20^{\circ}$ apart in phase) were stored using a high-resolution analog I/O board (National Instruments NB-A2100) in the computer (Apple Macintosh PowerPC).

\section{Data analysis}

From each series of 18 images, displacement paths within the imaging plane could be reconstructed for any selected structure by applying an optical flow algorithm based on spatial and temporal intensity gradients (Lucas and Kanade 1981; Barron et al. 1994; Hu 1999; Hu et al. 1999; Cai et al. 2003). Computation of displacement amplitude proceeded as follows: (1) a spatial bandpass filter was applied to each of the 18 images to increase the signal-to-noise ratio, (2) spatio-temporal derivatives were computed in $5 \times 5$ pixel neighborhoods, yielding frame-to-frame displacement vectors throughout the image area, (3) the displacement vectors at a given location were concatenated to yield a full cycle of motion for each structure of interest, and (4) the transverse amplitude component (defined as perpendicular to the reticular lamina) was computed for each displacement path (typically a linear back and forth motion or a small 
oval). Specifically, transverse displacement amplitudes were computed at each stimulus frequency for the tip of the glass paddle and for the basilar membrane at its upper marginal band near the base of the third

a

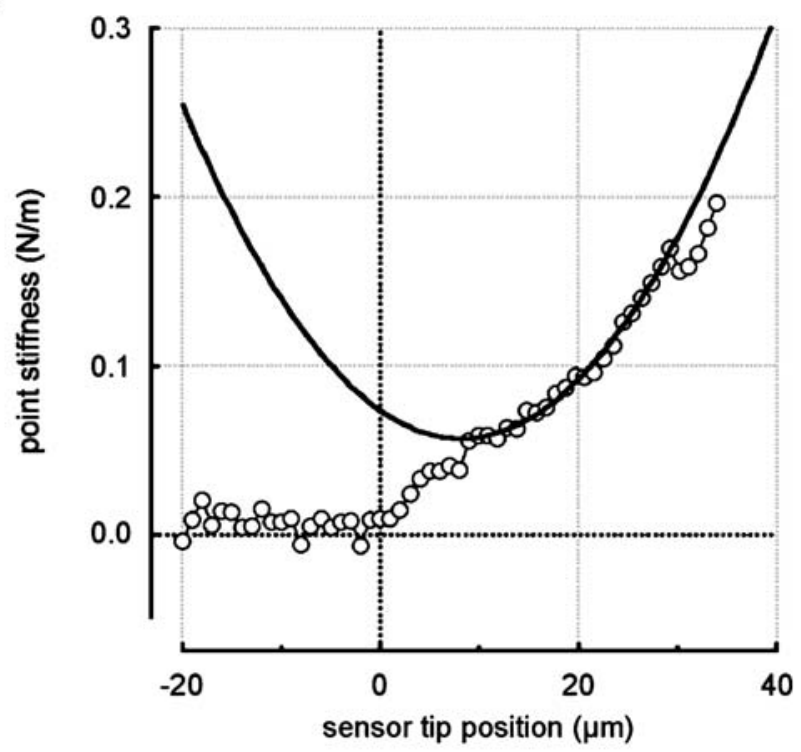

b

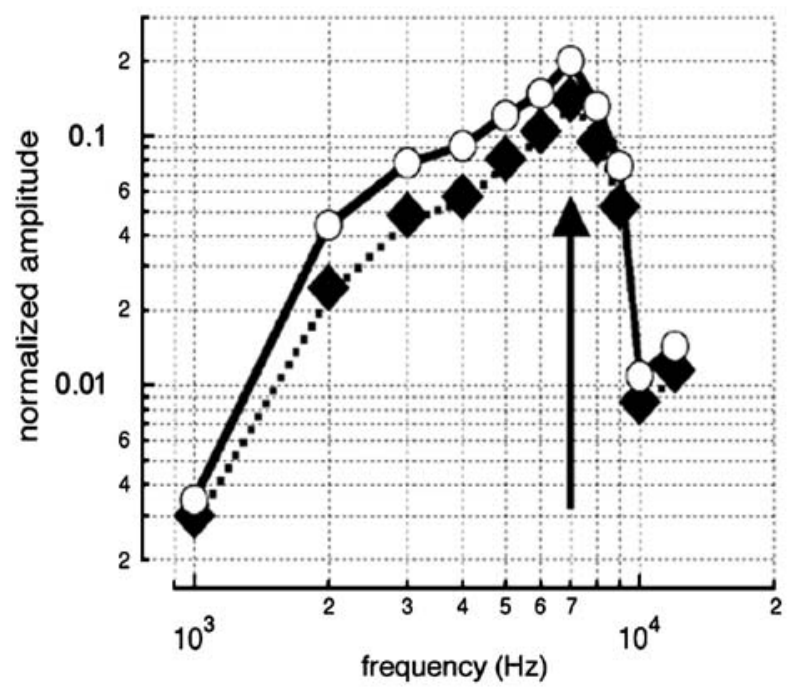

C

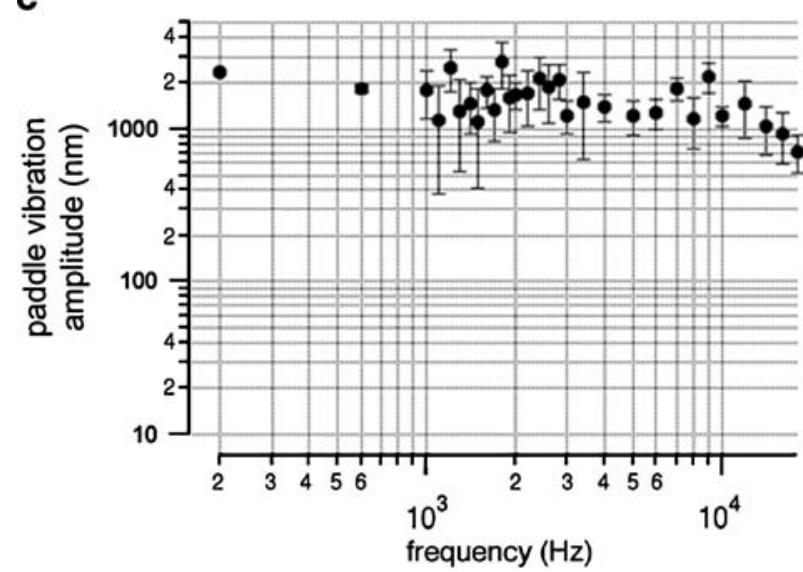

row of Deiters' cells. The ratio of basilar membrane to paddle amplitude was used to construct a frequency response curve. Best frequency (BF) was defined as the stimulus frequency yielding the maximum on this frequency response curve (Fig. 1b).

\section{System calibration}

Our motion measurement system was calibrated against the known displacements of a rigid probe attached to a precision piezo-electric linear actuator (Burleigh PZ-30). The probe displacement was independently determined by the voltage applied to the linear actuator $(5 \mu \mathrm{m} / 1,000 \mathrm{~V})$ and by the use of a calibration slide. The system noise floor was $100 \mathrm{~nm}$, and the basilar membrane displacement amplitudes were on the order of 500 to $1,000 \mathrm{~nm}$. The frequency response of the probe showed several maxima and minima (i.e., was not flat). During the experiments, we compensated for the frequency response of the actuator system by manually adjusting the driver voltage to obtain a relatively constant paddle displacement at every stimulus frequency. As an additional precaution, the actual paddle displacement was measured for all experiments so that a normalized basilar membrane displacement amplitude could be computed. The peak-to-peak amplitude of the paddle motion was typically $1.6 \mu \mathrm{m}$ (Fig. 1c).

\section{Cautionary points}

Although the video flow technique used here is a powerful method, it also possesses some drawbacks. Barron et al. (1994) compared different video flow techniques to determine which method is the most reliable in computing "optical flow" (or image velocity) from a sequence of images. One measure of reliability was the angular error (Fleet and Jepson 1990). Barron et al. found the expected error for the technique used here to be $0.21^{\circ}$. More recently, the

FIG. 1. a Example of stiffness of basilar membrane mid-pectinate site as a function of sensor tip position. Point stiffness (in $\mathrm{N} / \mathrm{m}$ ) is shown on the $y$-axis. The $x$-axis represents the position of the sensor tip (in $\mu \mathrm{m}$ ) as it is moved toward and onto the tissue: initial contact with the tissue occurs at $0 \mu \mathrm{m}$. The measurement shown was obtained at the middle turn location from a 13-day-old animal. The curve has been fitted with the following quadratic function to obtain a plateau stiffness value of $0.057 \mathrm{~N} / \mathrm{m}: k=0.057+0.00025(x-$ $8.2)^{2}$. b Determination of best frequency. The basilar membrane (open circles) and pillar foot (filled diamonds) vibrations have been normalized to the paddle vibration and plotted as a function of the stimulus frequency. The frequency at the maximum (arrow) was designated as the best frequency (BF) for the structure. c Amplitude of the paddle vibration at different stimulus frequencies. Shown is the average \pm the standard error across all experiments. Note that for any given experiment, the actual paddle vibration amplitude from that particular experiment was used to compute the ratio between the basilar membrane vibration and the paddle vibration. 
performance of the method was reinvestigated with data from experiments in the hemicochlea (Cai et al. 2003). The results showed an average absolute angular error of $14.9-21.3^{\circ}$ and an average vector magnitude error of 0.47 to 0.57 pixels, which corresponds to $169-196 \mathrm{~nm}$. An additional assumption in our technique is that the fluid layer that couples force from the paddle to the basilar membrane acts primarily as an inertial load, resulting in some possible low-pass filtering of the applied force (relative to the paddle displacement). Lee and Wen (2002) have examined the reactive force under conditions of oscillatory squeezing for an electrorheological fluid. With no applied voltage, this latter fluid behaves as a Newtonian fluid. They found that incorporating inertia into their model yields only a small effect on the amplitude of the reactive force (increasing slightly with frequency), with a somewhat larger effect on the phase lag of the reactive force. Because we do not expect any resonances in the amplitude component of the frequency response of the coupling between the paddle and the basilar membrane, our estimate of BF should not be confounded by the behavior of the fluid.

\section{Measurement of basilar membrane stiffness (piezoelectric sensor)}

Use and calibration of the stiffness measurement system have been described in detail (Emadi et al. 2004). The sensor consisted of a solid steel needle attached to a piezoelectric bimorph, designated as the sensor bimorph. This bimorph was attached in turn to a second piezoelectric bimorph, designated as the driver bimorph. During a stiffness measurement, the needle tip was positioned in contact with the basilar membrane, and the driver bimorph was used to deliver low-frequency $(10 \mathrm{~Hz})$ motion to the sensor bimorph and needle. The resulting sensor bimorph flexion, as manifested by a measurable voltage change, was used as an indicator of the load at the needle tip. The sensor system was calibrated against flexible glass test fibers, which were calibrated in turn on a "string instrument" (Zwislocki and Cefaratti 1989). Data were obtained from the basal and middle turns of hemicochleae mounted on the stage of an upright microscope (Leitz Ergolux AMC) sitting on a vibration isolation table (Newport VW-3646-OPT). Stiffness was measured as a function of tissue deflection at the middle of the basilar membrane pectinate zone (approaching from the scala tympani side) at least $100 \mu \mathrm{m}$ away from the cut edge of the hemicochlea. Stiffness-deflection curves of this type were fitted with a quadratic function (Fig. 1), based on a parallel beam model for the basilar membrane. The constant term from this fit was taken as the value for the "plateau" stiffness, which has been argued to represent the physiologically relevant stiffness of the basilar membrane (Gummer et al. 1981; Miller 1985; Olson and Mountain 1994). Because of the relatively short duration of these experiments (less than 2 hours), the material properties and structural relationships of the cellular and noncellular components of the hemicochlea are expected to remain constant. We have validated this assertion in a previous report (Emadi et al. 2004), based not only on the constancy of repeated stiffness measurements obtained in a hemicochlea over an extended time period but also on the equivalence of hemicochlea stiffness data and in vivo stiffness data.

\section{Statistical analysis}

Best frequency and basilar membrane stiffness were measured in the basal turn $(2.9 \pm 0.6 \mathrm{~mm}$ from the basal end of the basilar membrane) and in the middle turn $(7.3 \pm 0.4 \mathrm{~mm}$ from the basal end of the basilar membrane) of the gerbil hemicochlea preparation at ages spanning the onset and maturation of hearing: postnatal days 12, 13, 14, 15,16, 18, and older than 30 ; for the stiffness measurements, data points were obtained also at 10 and 11 days postnatal. Significant changes of either stiffness or best frequency during maturation were detected by performing a one-way analysis-of-variance for each measurement location, using the age of the gerbil as the treatment and a $99 \%$ criterion for significance. If a significant overall change with age was found for stiffness or BF, pairwise comparisons were made between individual ages using a Tukey-honestly-significant-difference test with $95 \%$ and $99 \%$ criteria for "significance" and "high significance", respectively.

\section{RESULTS}

\section{Developmental time course of best frequency}

Cochlear motion was elicited in the hemicochlea preparation with local mechanical stimulation, and the frequency response within a cross-section was measured. As previously demonstrated (Richter and Dallos 2003), the basilar membrane best frequency was always the same as that of other structures in a given cross-section. Shown in Figure 2 are representative tuning curves obtained from the basilar membrane vibration from different animals for different ages. Best frequency was determined for each of the curves and is shown in Figure 3 as a function of age for the basal and middle turn locations.

At the basal turn location, the best frequency is $4.9 \pm 1.7 \mathrm{kHz}$ at 12 and 13 days after birth (hearing onset) and then increases to reach $9.8 \pm 1.0 \mathrm{kHz}$ by 30 days after birth (mature hearing). This 1.0 octave developmental shift of BF seen in the hemicochlea is 

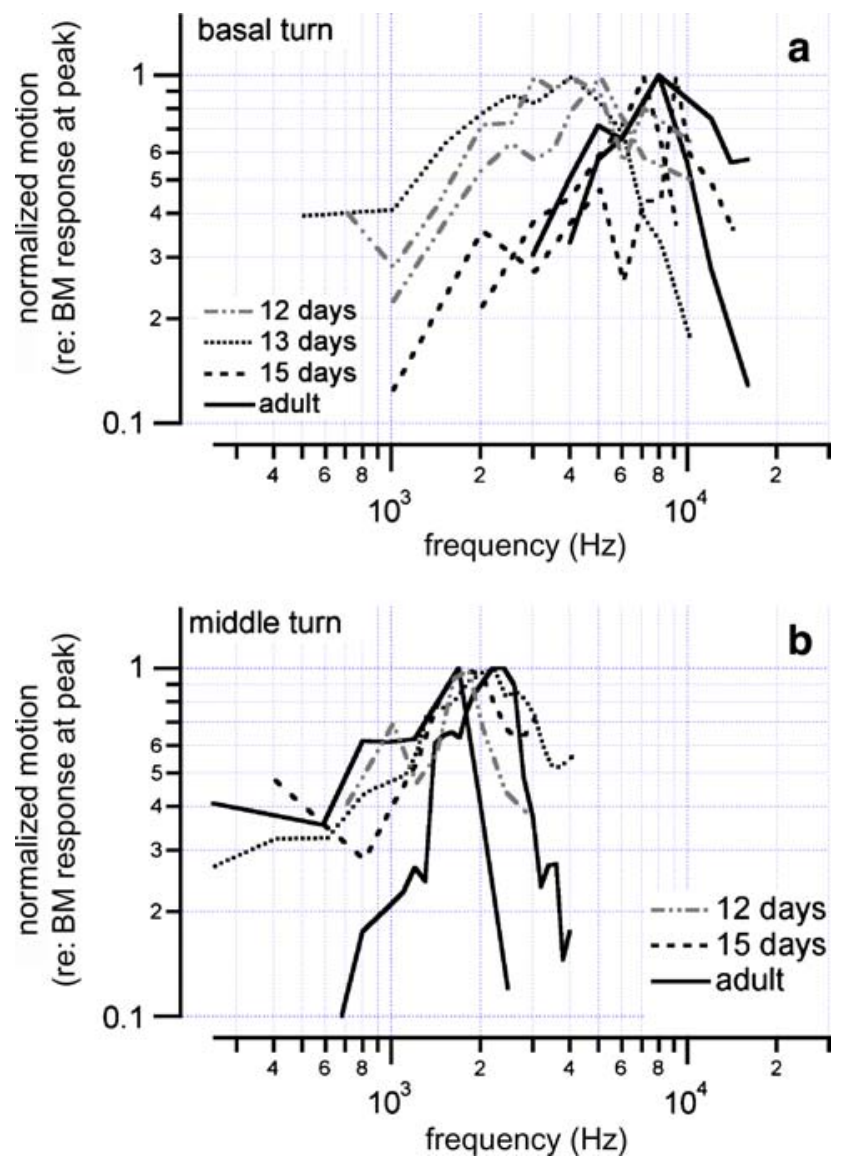

FIG. 2. Representative tuning curves computed for the basilar membrane in animals of different ages. a Data obtained from the basal turn. For a given curve, each point was computed as the ratio of basilar membrane to paddle displacement, and then the entire curve was normalized to the value at its peak. Note that the best frequencies (peaks of the tuning curves) shift from lower frequencies to higher frequencies with increasing age of the animals. b Data obtained from the middle turn. No systematic shift of best frequency can be seen with increasing age of the animals.

statistically significant and is consistent with previous observations on development of frequency response in the base of the gerbil cochlea (Harris and Dallos 1984; Yancey and Dallos 1985; Arjmand et al. 1988; Echteler et al. 1989). At the middle turn location, the best frequency is $1.7 \pm 0.3 \mathrm{kHz}$ at 12 days after birth and is $1.8 \pm 0.5 \mathrm{kHz}$ at 30 days and beyond. This 0.08 octave shift to higher best frequencies is not statistically significant, and is consistent with previous work showing no shift of best frequency with age in the middle turn (Arjmand et al. 1988; Müller 1996).

Developmental time course of basilar membrane stiffness

Figures 4 and 5 show point stiffness measurements taken with a piezoelectric sensor at the mid-pectinate zone of the basilar membrane in the gerbil hemi- cochlea preparation. The upper panels in Figure 4 show point stiffness from multiple ages plotted on a log-linear scale, and the lower panels show the same stiffness data plotted on a linear-linear scale. The panels on the left show data for the basal turn, and the panels on the right show data for the middle turn. Because many of the middle turn traces, especially for the younger ages, are close to the noise floor, Figure 5 contains an expanded view of the data from Figure $4 d$. Each panel in Figure 5 shows data from one of four representative ages $(11,12$, and 14 days after birth, and adult).

Plateau stiffness for the basal and middle turns are plotted as a function of age in Figure 6. Figure 7 summarizes our analysis of variance for the stiffness data, with age as the treatment. For the basal turn, the stiffness is $0.13 \mathrm{~N} / \mathrm{m}$ at 12 days after birth and increases to $0.73 \mathrm{~N} / \mathrm{m}$ by 18 days. The most significant change within a single 24-hour period occurs between 14 and 15 days, from 0.17 to $0.36 \mathrm{~N} / \mathrm{m}$. By 30 days, the stiffness is $0.78 \mathrm{~N} / \mathrm{m}$, which is not significantly different from that measured at 18 days. The change in stiffness by a factor of 5.6 for the basal turn between 12 and 18 days is statistically significant at the 99\% level.

For the middle turn, the mean stiffness at 12 days after birth is $0.050 \mathrm{~N} / \mathrm{m}$ and in the adult animals (>30 days) is $0.079 \mathrm{~N} / \mathrm{m}$. This change in stiffness by a factor of 1.6 between 12 days and adult is not statistically significant. The differences are, however, statistically significant (at the 99\% level) between postnatal day 11 and postnatal day 16 and between postnatal day 11 and adult. Note that because our data at day 11 are very close to the noise floor of our mea-

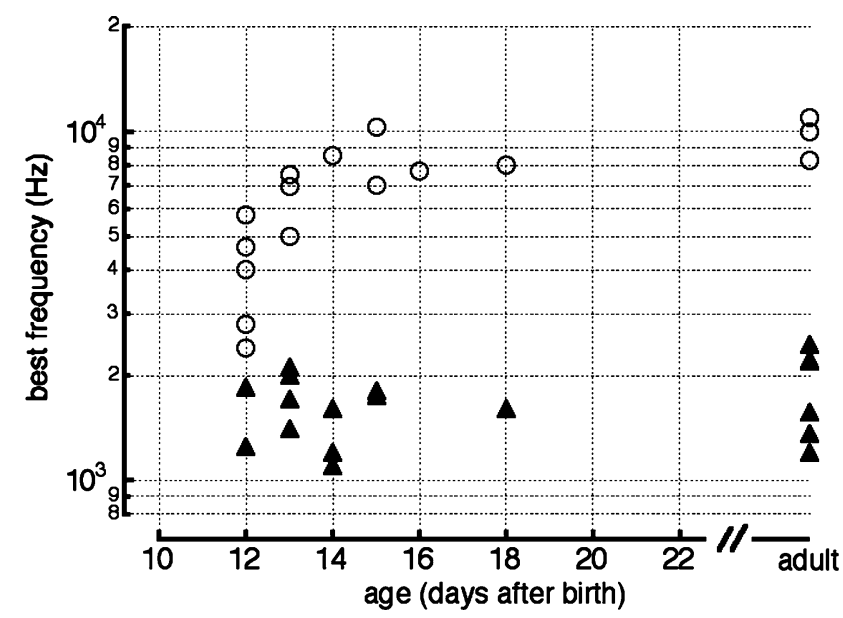

FIG. 3. Best frequency (BF) as a function of age. BFs were derived using the methodology shown in Figure 1b. Data are shown for the basal turn location (open circles) and for the middle turn location (filled triangles). 


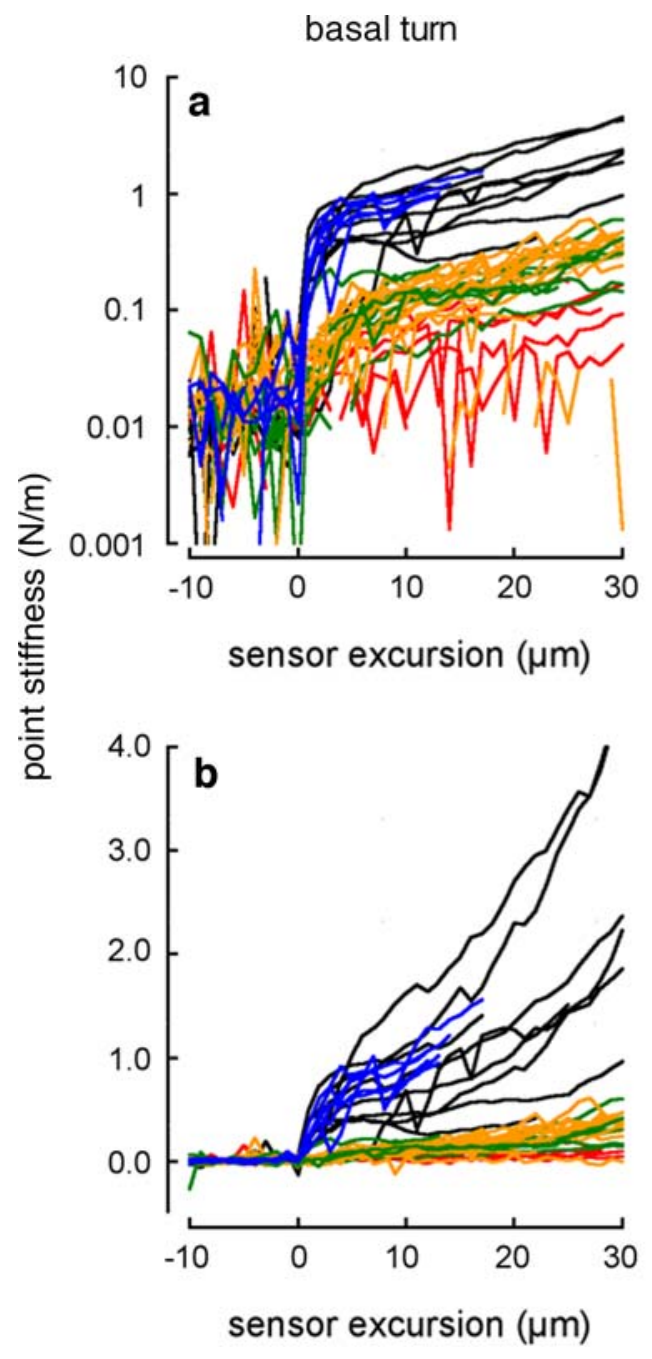

FIG. 4. Representative point stiffness traces for various ages. a Stiffness data for the basal turn location shown on a log-linear scale. b Stiffness data for the basal turn location shown on a linear-linear

surement system, our reported stiffness values for day 11 may actually be skewed higher than the real values.

In general, the variability in our middle turn data for any given age (see Figs. 4 and 5) suggests that the conclusions from our statistical analysis must be taken with some caution. There is the possibility that real developmental changes have been obscured by the variability in the stiffness data. As discussed in the Methods section, with regard to the general quality of each preparation, data were included for analysis only if a given hemicochlea met strict morphological criteria. A small portion of the measured variability in stiffness might be accounted for by the variation of longitudinal locations across hemicochleae. The location of the middle turn has been measured to be 7.3 $\mathrm{mm}$ from the basal end of the cochlea with a standard deviation of $0.4 \mathrm{~mm}$ (Edge et al. 1998). Using a value of $4.43 \mathrm{~dB} / \mathrm{mm}$ for the longitudinal stiffness gradient

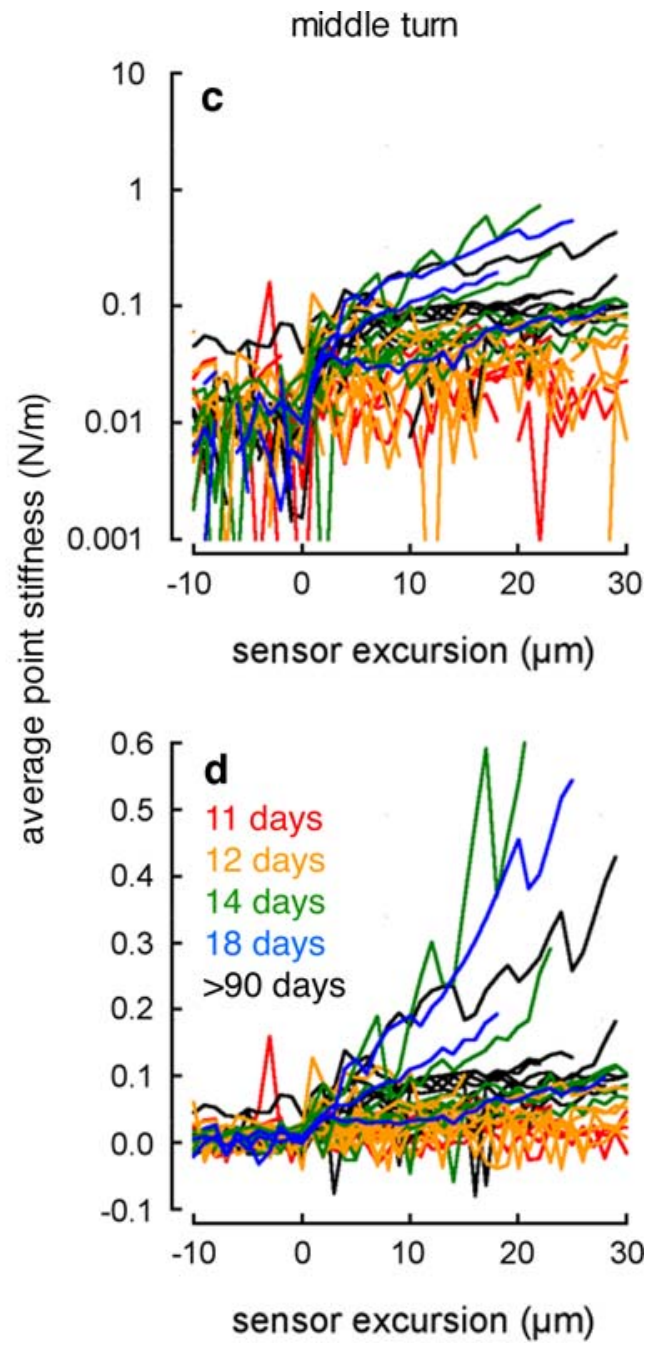

scale. c Stiffness data for the middle turn location shown on a loglinear scale. $\mathbf{d}$ Stiffness data for the middle turn location shown on a linear-linear scale.

in the adult gerbil cochlea (Emadi et al. 2004), the expected change of stiffness across $0.4 \mathrm{~mm}$ is $1.77 \mathrm{~dB}$ for the adult. In younger animals, assuming that the longitudinal gradient is shallower, the expected change of stiffness will be even smaller. An additional potential source of variability across measurements is radial placement of the sensor tip. All measurements reported here were taken nominally at the "midpectinate" position, but small deviations from this position could have had an effect on the measured stiffness traces (Emadi et al. 2004).

An additional observation for the basal turn stiffness data (Fig. 4a,b) is that the distance from the initial contact between the stiffness sensor and the tissue (zero on the $x$-axis) to the start of the plateau region appears to decrease with age. A first-order hypothesis for this phenomenon is that, for the younger ages, the first several microns of contact between the sensor 


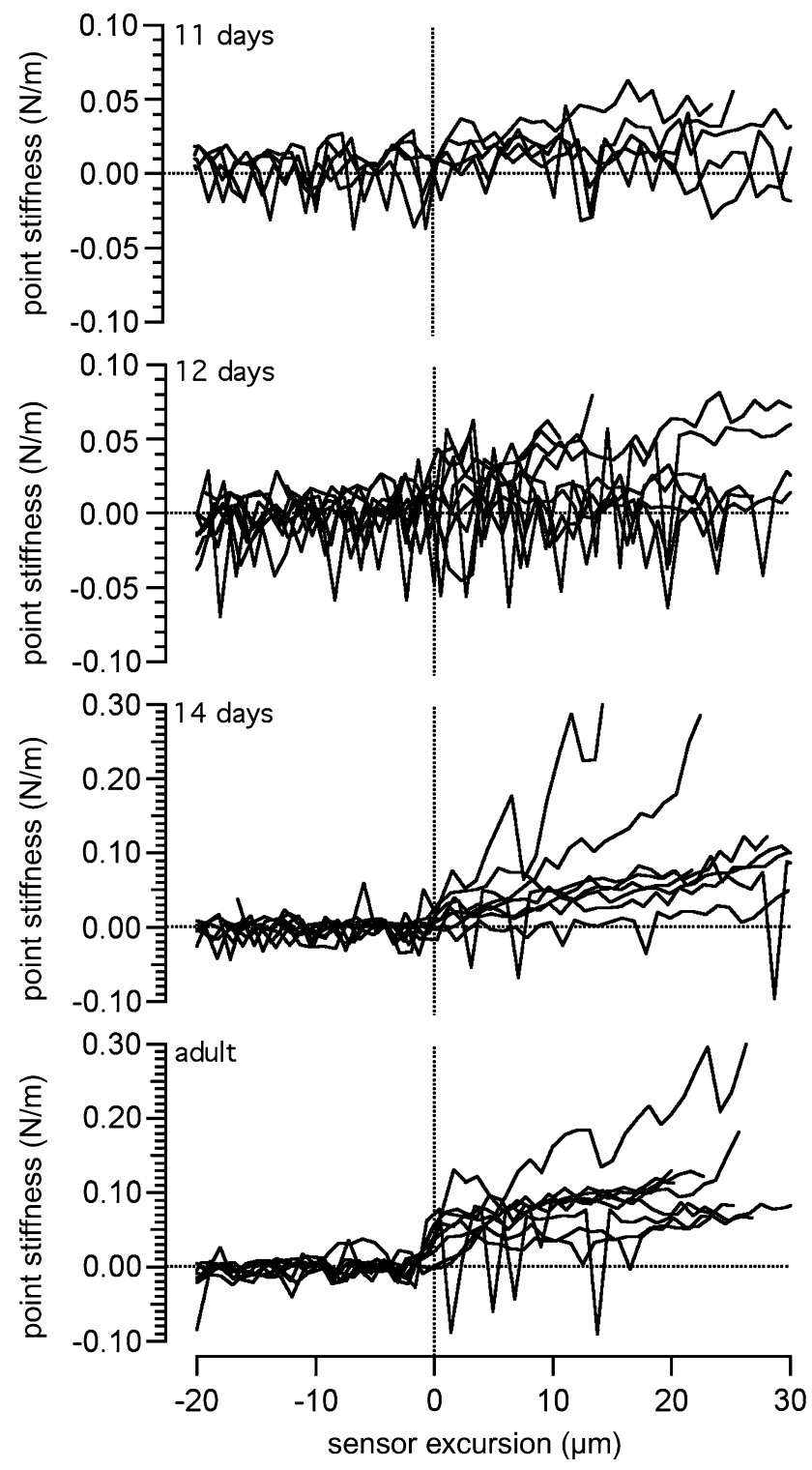

FIG. 5. Expanded view of representative data from Figure 4d. Each panel contains data obtained from the middle turn for a single age group.

and the tissue are dominated by the presence of the hyaline mass layer on the scala tympani side of the basilar membrane. This layer is present at early ages and then thins out by approximately 18 days after birth (Richter et al. 2000). Whereas this hypothesis may be valid, it is important to remember that, for our analysis, the initial contact between the sensor and the tissue is estimated post hoc by visual inspection of the measured data traces. This somewhat subjective assignment of the zero point on the $x$-axis complicates a proper analysis of the depth to the beginning of the plateau region. Note that any offset of the stiffness data along the $x$-axis does not affect our estimate of the plateau stiffness: this latter quantity is computed as the $y$-value at the center of a parabola fit to the rising portion of the stiffness trace, and this fitted parabola takes into account an arbitrary offset along the $x$-axis.

\section{DISCUSSION}

Comparison of adult data to previous work

The best frequencies $(\mathrm{BF})$ determined for the adult gerbil hemicochleae in the present study are approximately $0.5-1$ octaves below the characteristic frequencies (CFs) reported for adult gerbil cochleae in vivo (Müller 1996). This difference is as expected, keeping in mind that killing the animal immediately decreases the most sensitive frequency by approximately $0.6-$ 0.8 octaves (Rhode 1974; Rhode and Robles 1974; Ruggero and Rich 1991). An additional comparison to the in vivo data can be made by examining the ratio of the $\mathrm{BF}$ in the basal turn to that in the middle turn. In the present experiments, the $\mathrm{BF}$ decreases from the basal turn to the middle turn by a factor of 5.4 (from 9.8
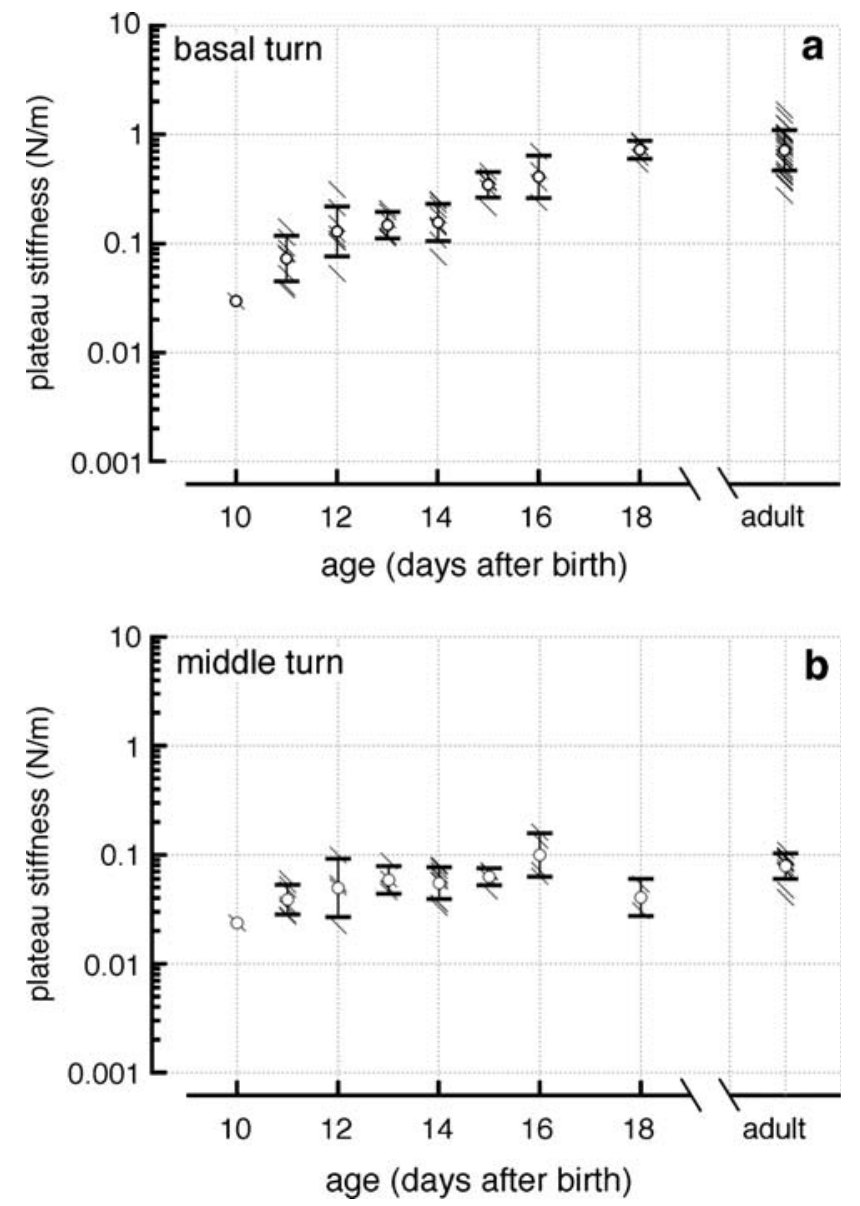

FIG. 6. Plateau stiffness values as a function of age. Data are shown for the basal turn location (panel a) and for the middle turn location (panel b). Slanted bars are individual measurements. Open circles are the corresponding averages \pm the standard deviation. 


\begin{tabular}{|c|c|c|c|c|c|c|c|c|c|c|c|c|}
\hline \multicolumn{13}{|c|}{ Basal Turn } \\
\hline \multirow[b]{2}{*}{ Age } & \multirow[b]{2}{*}{$N$} & \multirow{2}{*}{$\begin{array}{l}\text { Average } \\
\text { (dB re: } \\
1 \mathrm{~N} / \mathrm{m}) \\
\end{array}$} & \multirow{2}{*}{$\begin{array}{c}\text { Average } \\
\text { (N/m) }\end{array}$} & \multirow{2}{*}{$\begin{array}{c}\text { Variance } \\
(d B)\end{array}$} & \multicolumn{8}{|c|}{ Statistically different from.. } \\
\hline & & & & & adult & $18 d a b$ & $16 d a b$ & $15 d a b$ & $14 d a b$ & $13 d a b$ & $12 d a b$ & $11 d a b$ \\
\hline 11 dab & 8 & -22.8 & 0.072 & 17.4 & $\star \star$ & $\star \star$ & $\star \star$ & $\star \star$ & $\star \star$ & & * & \\
\hline 12 dab & 8 & -17.8 & 0.129 & 21.2 & $\star \star$ & $\star \star$ & $\star \star$ & $\star \star$ & & & & * \\
\hline 13 dab & 6 & -16.7 & 0.147 & 6.0 & $\star \star$ & $\star \star$ & $\star \star$ & * & & & & \\
\hline 14 dab & 11 & -16.1 & 0.157 & 11.4 & $\star \star$ & $\star \star$ & $\star \star$ & *夫 & & & & $\star \star$ \\
\hline 15 dab & 6 & -9.2 & 0.346 & 5.3 & $\star \star$ & * & & & $\star \star$ & * & $\star \star$ & $\star \star$ \\
\hline 16 dab & 4 & -7.8 & 0.409 & 15.4 & & & & & $\star \star$ & ** & $\star \star$ & ** \\
\hline 18 dab & 6 & -2.8 & 0.726 & 2.8 & & & & * & $\star \star$ & $\star \star$ & $\star \star$ & $\star \star$ \\
\hline adult & 34 & -2.9 & 0.715 & 13.7 & 2 & & & $\star \star$ & $\star \star$ & $\star \star$ & $\star \star$ & $\star \star$ \\
\hline
\end{tabular}

\begin{tabular}{|c|c|c|c|c|c|c|c|c|c|c|c|c|}
\hline \multicolumn{13}{|c|}{ Middle Turn } \\
\hline \multirow[b]{2}{*}{ Age } & \multirow[b]{2}{*}{$N$} & \multirow{2}{*}{$\begin{array}{l}\text { Average } \\
\text { (dB re: } \\
1 \mathrm{~N} / \mathrm{m}) \\
\end{array}$} & \multirow{2}{*}{$\begin{array}{c}\text { Average } \\
(\mathrm{N} / \mathrm{m})\end{array}$} & \multirow{2}{*}{$\begin{array}{c}\text { Variance } \\
(d B)\end{array}$} & \multicolumn{8}{|c|}{ Statistically different from.. } \\
\hline & & & & & adult & $18 d a b$ & $16 d a b$ & $15 \mathrm{dab}$ & $14 d a b$ & $13 d a b$ & $12 d a b$ & $11 d a b$ \\
\hline $11 \mathrm{dab}$ & 7 & -28.2 & 0.039 & 7.6 & $\star \star$ & & $\star \star$ & & & & & \\
\hline $12 \mathrm{dab}$ & 4 & -26.0 & 0.050 & 28.3 & & & & & & & & \\
\hline 13 dab & 4 & -24.7 & 0.059 & 6.5 & & & & & & & & \\
\hline $14 \mathrm{dab}$ & 10 & -25.2 & 0.055 & 8.6 & & & & & & & & \\
\hline 15 dab & 5 & -24.0 & 0.063 & 2.5 & & & & & & & & \\
\hline $16 \mathrm{dab}$ & 4 & -20.0 & 0.100 & 16.0 & & & & & & & & $\star \star$ \\
\hline $18 \mathrm{dab}$ & 2 & -27.9 & 0.040 & 12.0 & & & & & & & & \\
\hline adult & 15 & -22.1 & 0.079 & 5.6 & 2 & & & & & & & $\star \star$ \\
\hline
\end{tabular}

FIG. 7. Summary statistics and analysis of variance (ANOVA) for basal turn and middle turn. The upper table shows data for the basal turn, and the lower table shows data for the middle turn. The left half of each table provides summary statistics with columns for age, number of samples, average stiffness in $\mathrm{dB}$ re: $1 \mathrm{~N} / \mathrm{m}$, average stiffness in $\mathrm{N} / \mathrm{m}$, and variance in $\mathrm{dB}$. The right half of each table summarizes the results from an ANOVA, using age as the treatment. Single asterisks indicate a significant difference between two ages at a $95 \%$ confidence level, and double asterisks indicate a significant difference between two ages at a 99\% confidence level. to $1.8 \mathrm{kHz}$ ), similar to the decrease of $\mathrm{CF}$ by a factor of 6 (from 18 to $3 \mathrm{kHz}$ ) observed for the in vivo preparation. Note that in previous work on the hemicochlea, BFs were reported to be approximately 1.4 octaves below the in vivo CFs and the ratio of BFs between basal and middle turn locations was reported to be a factor of 3. There are two important changes that may account for the overall higher BFs and the more in vivo-like $\mathrm{BF}$ ratio in the present study compared to the previous hemicochlea study: (1) the time between euthanizing the animal and the first measurement is significantly shorter (by more than 30 minutes) in the new experiments, and (2) the composition of the bath solution has been improved to maintain the integrity of the preparation for up to 2 hours, an increase of more than 90 minutes beyond the previous study.

With regard to the basilar membrane stiffness data in the present study, the values decrease from the basal turn to the middle turn by a factor of 9.1 in adult animals. This longitudinal stiffness ratio is larger than the factor of 3 derived from the data reported by Naidu and Mountain (1998) for the equivalent distance along their in vitro adult gerbil basilar membrane preparation. Possible reasons for the discrepancies between the two sets of stiffness data have been discussed previously (Emadi et al. 2004).

\section{Comparison of best frequency developmental data to previous work}

Our best frequency developmental data are in good agreement with other findings reported for the gerbil. For example, Müller (1996) recorded from single auditory nerve fibers between the onset of hearing (postnatal day 12) and postnatal day 18 and found a developmental shift of best frequency by a factor of 1.9 (0.9 octaves) for a location corresponding to our basal turn location (2.9 $\mathrm{mm}$ from the basal end of the basilar membrane) and by a factor of 1.29 (0.4 octaves) for the location corresponding to our middle 
turn location (7.3 $\mathrm{mm}$ from the basal end of the basilar membrane). Arjmand et al. (1988) recorded cochlear microphonics from the round window of the gerbil cochlea between postnatal day 12 and postnatal day 20 and reported frequency shifts of approximately 1.5 octaves for the mid-basal turn and 0.14 octaves for the middle turn. Echteler et al. (1989) recorded from spiral ganglion cells of the gerbil cochlea between postnatal day 14 and postnatal day 20 and reported a shift in best frequency by 1.5 octaves in the mid-basal turn. Harris and Dallos (1984) measured cochlear microphonics in gerbils between postnatal day 12 and adult ( $>30$ days) and found an increase in best frequency of approximately 2 octaves. Overstreet et al. (2002) measured the vibration amplitude of the gerbil basilar membrane at $1.2 \mathrm{~mm}$ from the basal end between postnatal day 14 and adult and reported an increase of best frequency by 1.7 octaves. Finally, Mills and Rubel (1998) measured distortion product emissions before and after furosemide injection to assess the gain of the active processes within the cochlea. They reported that the cut-off frequency of the gain function increased by approximately two octaves between postnatal day 15 and postnatal day 28 .

\section{Relationship between stiffness} and BF during development

The stiffness and BF data can be quantitatively related by modeling a given local segment of the cochlea as a simple resonance, such that resonant frequency $=$ (stiffness / mass) ${ }^{0.5}$ (Emadi et al. 2004). Assuming that tissue density remains constant, changes in effective mass are approximated by changes in cross-sectional area of the tissue. For our basal turn measurement location between 12 days after birth and adult (>30 days), the basilar membrane mid-pectinate point stiffness increases by a factor of 5.5 and the combined cross-sectional area of the cochlear tissues (basilar membrane, organ of Corti, and tectorial membrane) increases by a factor of 1.1 (from 7,870 to $8,630 \mu \mathrm{m}^{2}$; Richter et al. 2000). We include the organ of Corti and the tectorial membrane in the cross-sectional area because it is assumed that the mechanical response of the basilar membrane is affected by the attached structures. Similarly, the point stiffness measured at the basilar membrane is presumed to include contributions from the attached structures. Incorporating these data into the spring-mass resonance model, the measured stiffness and area changes yield an expected increase of resonant frequency by a factor of 2.24 (1.16 octaves), which is similar to the factor of 2 (1.0 octaves) increase of BF measured for the basal turn. This simple analysis demonstrates that developmental stiffness and mass changes can account for changes of the basilar membrane frequency response, with an error of only 0.16 octaves.

Similar calculations were made for the middle turn location. Although the results of our ANOVA suggest that, in terms of basilar membrane stiffness, postnatal day 11 is the only age that is significantly different from the adult, we do not have best frequency data for postnatal day 11. With that limitation in mind, we present the resonant frequency analysis comparing 12 days after birth and adult. The basilar membrane midpectinate point stiffness increases by a factor of 1.6 between 12 days after birth and adult (>30 days), and the combined cross-sectional area of the cochlear tissues (basilar membrane, organ of Corti, and tectorial membrane) increases by a factor of 1.1 (from 30,166 to $34,080 \mu^{2}$; Richter et al. 2000). Incorporating these data into the spring-mass resonance model, the measured stiffness and area changes yield an expected increase of resonant frequency by a factor of 1.3 (0.4 octaves) for the middle turn between 12 days and adult. As stated earlier, the measured change in best frequency between these ages is 0.1 octaves, but is not statistically significant.

In our previous work in the adult gerbil hemicochlea (Emadi et al. 2004), we noted that although absolute values of point stiffness and tissue mass might be scaled by some unknown factor, the measurements provided a useful indication of spatial gradients of these mechanical parameters along the length of the cochlea. In a similar vein, we contend that the measurements presented here provide an accurate depiction of temporal gradients of the mechanical properties of the gerbil cochlea over the course of development.

\section{Relative developmental contributions of active processes versus passive mechanics}

We argue that, after the onset of hearing (at 12 days after birth), little if any of the developmental shift of BF measured in the basal or middle turn is the result of changes in the active mechanics of the cochlea. We base this argument on observations that the so-called cochlear amplifier (which yields sharpened frequency selectivity, more sensitive tuning curves, and a nonlinear increase in vibration amplitude with increasing sound level) is already functional (Norton et al. 1991) and that the outer hair cells (thought to be an essential component of the cochlear amplifier) are already motile (He et al. 1994) by postnatal day 12. More significantly, measurements of basilar membrane vibrations made in preparations in which the cochlear amplifier is nonfunctional have shown that most of the developmental BF shift is brought about by changes in passive mechanics (Overstreet and Ruggero 1999; Richter and Dallos 2000; Overstreet et al. 2002). 


\section{ACKNOWLEDGMENTS}

This work was supported by the National Science Foundation (IBN-077476 and IBN-0415901), the American Hearing Research Foundation, and the National Institute for Deafness and Other Communication Disorders (DC-00708). GE was supported in part by a grant from the Hugh Knowles Center at Northwestern University. The authors thank Dr. Peter Dallos for providing equipment and support to accomplish the experiments and for feedback on the manuscript. The image acquisition software for the motion measurements was written by Xintian $\mathrm{Hu}$.

\section{REFERENCES}

Arjmand E, Harris D, Dallos P. Developmental changes in frequency mapping of the gerbil cochlea: comparison of two cochlear locations. Hear Res. 32 1:93-96, 1988.

Barron JL, Fleet DJ, Beauchemin SS. Performance of optical flow techniques. Int. J. Comp. Vis. 12 1:43-77, 1994.

Cai H, Richter CP, Chadwick RS. Motion analysis in the hemicochlea. Biophys. J. 85 3:1929-1937, 2003.

ECHTELER SM. Structural correlates of frequency-place map development. Abstr. Assoc. Res. Otolaryngol. 18:442, 1995.

Echteler SM, Arjmand E, Dallos P. Developmental alterations in the frequency map of the mammalian cochlea. Nature 341 6238:147-149, 1989.

Edge RM, Evans BN, Pearce M, Richter CP, Hu X, Dallos P. Morphology of the unfixed cochlea. Hear Res. 124 1-2:1-16, 1998.

Emadi G, Richter CP, Dallos P. Changes of stiffness in the gerbil cochlea after the onset of hearing. Abtsr. Assoc. Res. Otolaryngol. 24:97, 2001.

Emadi G, Richter CP, Dallos P. Stiffness of the gerbil basilar membrane: radial and longitudinal variations. J. Neurophysiol. 91 1:474-488, 2004.

Fleet DJ, JePSON AD. Computation of component image velocity from local phase information. Intern. J. Comput. Vis. 5:77-104, 1990.

Gummer AW, Johnstone BM, Armstrong NJ. Direct measurement of basilar membrane stiffness in the guinea pig. J. Acoust. Soc. Am. 70:1298-1309, 1981.

Harris DM, Dallos P. Ontogenetic changes in frequency mapping of a mammalian ear. Science. 225 4663:741-743, 1984.

He DZ, Evans BN, Dallos P. First appearance and development of electromotility in neonatal gerbil outer hair cells. Hear Res. 78 1:77-90, 1994.

Hu X. A computer vision study on cochlear micromechanics. Doctoral Dissertation Thesis, Northwestern University, Evanston, IL, 1999.

Hu X, Evans BN, Dallos P. Transmission of basilar membrane motion to reticular lamina motion. Abtsr. Assoc. Res. Otolaryngol., pp. 223, 1995.

Hu X, Evans BN, Dallos P. Direct visualization of organ of Corti kinematics in a hemicochlea. J. Neurophysiol. 82 5:2798-2807, 1999.

LEE CY, WEN CY. The oscillatory squeeze flow of electrorheological fluid considering the inertia effect. Smart Mater. Struct. 11:553-560, 2002.

LucAS B, KanADE T. An iterative image registration technique with an application to stereo vision. In Proceedings of the 1981 DARPA Image Understanding Workshop, pp. 121-130, 1981.

McGuirt JP, Schmiedt RA, Schulte BA. Development of cochlear potentials in the neonatal gerbil. Hear Res. 84:52-60, 1995.
Miller CE. Structural implications of basilar membrane compliance measurements. J. Acoust. Soc. Am. 77 4:1465-1474, 1985.

Mills DM, Rubel EW. Development of the cochlear amplifier. J. Acoust. Soc. Am. 100 1:428-441, 1996.

Mills DM, RuBEL EW. Development of distortion product emissions in the gerbil: 'filter' response and signal delay. J. Acoust. Soc. Am. 101 1:395-411, 1997.

Mills DM, Rubel EW. Development of the base of the cochlea: place code shift in the gerbil. Hear Res. 122 1-2:82-96, 1998.

MüLler M. The cochlear place-frequency map of the adult and developing Mongolian gerbil. Hear Res. 94 1-2:148-156, 1996.

NAidu RC, Mountain DC. Measurements of the stiffness map challenge a basic tenet of cochlear theories. Hear Res. 124 1-2:124-131, 1998.

Naidu RC, Mountain DC. Longitudinal coupling in the basilar membrane. J. Assoc. Res. Otolaryngol. 2 3:257-267, 2001.

Norton SJ, Bargones JY, Rubel EW. Development of otoacoustic emissions in gerbil: evidence for micromechanical changes underlying development of the place code. Hear Res. 51 1:73-91, 1991.

Olson ES, Mountain DC. Mapping the cochlear partition's stiffness to its cellular architecture. J. Acoust. Soc. Am. 95 1:395-400, 1994.

Overstreet EH, Ruggero MA. The development of the basilar membrane mechanics at the hook region of the Mongolian gerbil cochlea. Assoc. Res. Otolaryngol. 22:537, 1999 (Abstract).

Overstreet EH 3Rd, Temchin AN, Ruggero MA. Passive basilar membrane vibrations in gerbil neonates: mechanical bases of cochlear maturation. J. Physiol. 545 Pt 1:279-288, 2002.

Overstreet EH, Richter C-P, Temchin AN, Cheatham MA, Ruggero MA. High-frequency sensitivity of the mature gerbil cochlea and its development. Audiol. Neurootol. 8:19-27, 2003.

RHODE WS. Measurement of vibration of the basilar membrane in the squirrel monkey. Ann. Otol. Rhinol. Laryngol. 83 5:619625,1974

RHODE WS, RoBles L. Evidence from Mossbauer experiments for nonlinear vibration in the cochlea. J. Acoust. Soc. Am. 55 3:588-596, 1974.

Richter CP, Dallos P. Micromechanics contribute to the shift in the frequency place code in developing gerbils. Abtsr. Assoc. Res. Otolaryngol., 23:870, 2000.

Richter C-P, Dallos P. Micromechanics in the gerbil hemicochlea. In: Gummer AW (ed) Biophysics of the cochlea. World Scientific Publishing Co. Pte. Ltd., Singapore, pp. 278-284, 2003.

Richter CP, Evans BN, Edge R, Dallos P. Basilar membrane vibration in the gerbil hemicochlea. J. Neurophysiol. 79 5:2255-2264, 1998.

Richter CP, Edge R, He DZ, Dallos P. Development of the gerbil inner ear observed in the hemicochlea. J. Assoc. Res. Otolaryngol. 1 3:195-210, 2000.

RugGero MA, Rich NC. Furosemide alters organ of corti mechanics: evidence for feedback of outer hair cells upon the basilar membrane. J. Neurosci. 11 4:1057-1067, 1991.

Schweitzer L, Lutz C, Hobbs M, Weaver SP. Anatomical correlates of the passive properties underlying the developmental shift in the frequency map of the mammalian cochlea. Hear Res. 97:84-94, 1996.

Teudt IU, Richter CP. The hemicochlea preparation of the guinea pig and other mammalian cochleae. J. Neurosci. Methods, 2007.

Woolf NK, Ryan AF. The development of auditory function in the cochlea of the mongolian gerbil. Hear. Res. 13:277-283, 1984.

YANCEY C, DALlos P. Ontogenic changes in cochlear characteristic frequency at a basal turn location as reflected in the summating potential. Hear. Res. 18 2:189-195, 1985.

Zwislocki JJ, Cefaratti LK. Tectorial membrane. II: Stiffness measurements in vivo. Hear Res. 42 2-3:211-227, 1989. 